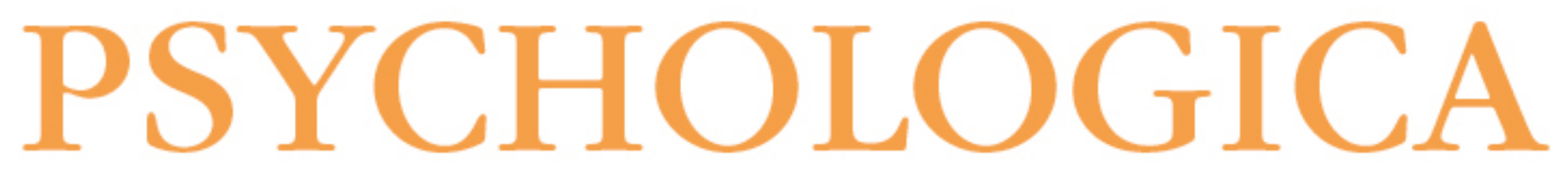

Falsas memórias e tempo de reação: estudo com o procedimento de palavras associadas

Autor(es): $\quad \begin{array}{ll}\text { Sene, Arthur Siqueira de; Lopes, Ederaldo José; Rossini, Joaquim } \\ \text { Carlos }\end{array}$

Publicado por: Imprensa da Universidade de Coimbra

URL

persistente: URI:http://hdl.handle.net/10316.2/35820

DOI: $\quad$ DOI:http://dx.doi.org/10.14195/1647-8606_57_1_2

Accessed : $\quad$ 26-Apr-2023 04:13:04

A navegação consulta e descarregamento dos títulos inseridos nas Bibliotecas Digitais UC Digitalis, UC Pombalina e UC Impactum, pressupõem a aceitação plena e sem reservas dos Termos e Condições de Uso destas Bibliotecas Digitais, disponíveis em https://digitalis.uc.pt/pt-pt/termos.

Conforme exposto nos referidos Termos e Condições de Uso, o descarregamento de títulos de acesso restrito requer uma licença válida de autorização devendo o utilizador aceder ao(s) documento(s) a partir de um endereço de IP da instituição detentora da supramencionada licença.

Ao utilizador é apenas permitido o descarregamento para uso pessoal, pelo que o emprego do(s) título(s) descarregado(s) para outro fim, designadamente comercial, carece de autorização do respetivo autor ou editor da obra.

Na medida em que todas as obras da UC Digitalis se encontram protegidas pelo Código do Direito de Autor e Direitos Conexos e demais legislação aplicável, toda a cópia, parcial ou total, deste documento, nos casos em que é legalmente admitida, deverá conter ou fazer-se acompanhar por este aviso. 
VOLUME $5 \longdiv { 2 0 1 4 }$

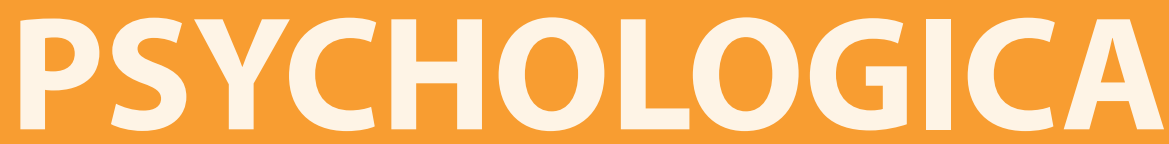

IMPRENSA DA UNIVERSIDADE DE COIMBRA

COIMBRA UNIVERSITY PRESS

FACULDADE DE PSICOLOGIA E DE CIÊNCIAS

DA EDUCAÇÃO DA UNIVERSIDADE DE COIMBRA 


\title{
Falsas memórias e tempo de reação: estudo com o procedimento de palavras associadas
}

\author{
Arthur Siqueira de Sene ${ }^{1}$, Ederaldo José Lopes² e Joaquim Carlos Rossini ${ }^{3}$
}

False memories and reaction times: a literature review and experimental data

\begin{abstract}
False Memories (FM) are based on memory errors and occur when people remember situations that never happened to them, events at which they were not present, or when remembrance from occurred facts are reminded differently from what really happened. This paper aims to present reaction time data about false memories with the adaptation of the DRM (Deese-Roediger-McDermott) paradigm into Brazilian Portuguese. The experiment was performed by 12 participants. Sets of memorized words (CPM) composed of 3, 5 or 7 items and the type of test (target, critical distractor and not related distractor) were manipulated within subjects. The results indicate a trend of increasing in reaction time according to the set of memorized words and confirm the importance of reaction time as a measure to study false memories in short-term situations.
\end{abstract}

Keywords: false memories; reaction time; DRM paradigm

\section{Resumo}

Falsas Memórias (FM) são baseadas em erros da memória e ocorrem quando as pessoas lembram-se de situações que não aconteceram, eventos não presenciados, ou então, as

1 Instituto de Psicologia da Universidade Federal de Uberlândia. Email: arthursene@yahoo.com.br

2 Instituto de Psicologia da Universidade Federal de Uberlândia.

3 Instituto de Psicologia da Universidade Federal de Uberlândia. 
lembranças dos fatos ocorridos são lembrados de maneira distorcida. O objetivo desse artigo é apresentar dados de tempo de reação sobre falsas memórias com a adaptação do paradigma DRM (Deese-Roediger-McDermott) para o português brasileiro. O experimento foi aplicado em 12 participantes. Foram manipulados os fatores conjuntos de palavras memorizadas (CPM) compostos por 3, 5 ou 7 itens e o tipo de prova (alvo, distrator crítico e distrator não relacionado). Os resultados apontam uma tendência de aumento do tempo de reação em função do conjunto de palavras memorizadas e confirmam a importância do tempo de reação como uma medida para se estudar as falsas memórias em situações de curto prazo.

Palavras-chave: falsas memórias; tempo de reação; procedimento DRM

\section{INTRODUÇÃO}

A memória pode ser conceituada como o meio pelo qual nós codificamos, armazenamos e recuperamos as informações passadas para usá-las no presente (Sternberg, 2008). No entanto, determinadas lembranças recuperadas podem ser feitas à custa de erros ou distorções. Entre eles destacam-se as falsas memórias (FM).

Roediger e McDermott (1995) referem-se às FM como lembranças de eventos que nunca ocorreram ou da recordação de um evento de uma maneira muito diferente daquela quando o evento ocorreu (cf. p. 803). Mazzoni e Scoboria (2007) afirmam que, em geral, as FM podem ser definidas como qualquer instância na qual a memória é reportada para um evento ou componente de um evento que não tenha sido experienciado. A dificuldade de distinção entre as FM e as memórias verdadeiras se deve ao fato de os indivíduos terem a capacidade de descrever alguma coisa em seus mínimos detalhes (Easenkraemer, 2006). Nem toda compreensão/interpretação de um evento resulta em falhas de memória; daí a importância de se estudar os elementos envolvidos na distinção entre memórias verdadeiras e memórias falsas, pois ela perpassa as mais diversas situações do nosso cotidiano.

O pioneiro nos estudos da falsificação de memórias com adultos foi o de Bartlett (1932). Ele mostrou a importância das expectativas individuais para o entendimento e a recordação dos fatos. Além disso, descreveu a recordação como um processo de reconstrução, que tem como base os esquemas mentais e o conhecimento geral prévio da pessoa, enfatizando, assim, o papel da compreensão e da influência cultural na lembrança (Neufeld, Brust, \& Stein, 2010; Stein \& Neufeld, 2001). Loftus (1997) ressaltou o caráter construtivo da memória mediante a combinação de memórias atuais com conteúdos sugestivos recebidos de outras pessoas, sendo que durante esse processo os indivíduos ficam suscetíveis ao esquecimento da fonte da informação. 
Num estudo clássico, Loftus e Palmer (1974) introduziram o procedimento de falsa informação, ou seja, logo após a experiência vivida foi apresentada uma informação compatível com a experiência, porém falsa. Os resultados produziram o efeito de falsa informação, isto é, os índices de reconhecimento verdadeiro diminuíram e os de reconhecimento falso tiveram um aumento significativo (Stein \& Perguer, 2001). O efeito da falsa informação tem a capacidade potencial de invasão de nossas memórias quando nós falamos com outras pessoas, quando somos inquiridos sugestivamente ou, mesmo, quando nós lemos ou vemos a cobertura pelos meios de comunicação social sobre algum evento que nós podemos ter experimentado (Loftus, 1997).

As FM podem se originar de duas formas: a forma espontânea e implantada ou sugerida. As FM espontâneas são criadas internamente como produto do processo normal de compreensão do evento realizado pelo indivíduo (Reyna \& Lloyd, 1997; Stein \& Perguer, 2001). As FM espontâneas são resultantes de distorções endógenas, frutos do próprio funcionamento da memória, livre de interferência de fonte externa (Neufeld et al., 2010). As FM sugeridas ou implantadas são aquelas relacionadas com o resultado de uma sugestão externa, independentemente de ser propositadamente ou não, e seu conteúdo não faz parte do evento que foi experienciado, apesar de conter características que têm coerência com o evento (Reyna \& Lloyd, 1997).

Três modelos teóricos têm servido de base para a compreensão das FM: o construtivista, o monitoramento da fonte e a teoria do traço difuso (TTD). Segundo Neufeld et al. (2010) no modelo construtivista a informação inicial é integrada em informações prévias que o indivíduo possui e com isso podem ocorrer distorções e sobreposições à memória inicial, o que pode gerar uma FM (Reyna \& Lloyd, 1997; Stein \& Neufeld, 2001). No monitoramento da fonte (Johnson, Hashtroudi, \& Lindsay, 1993), a geração das FM estaria localizada nas dificuldades em diferenciar a fonte verdadeira da memória recuperada e outras fontes, sejam elas internas pensamentos, imagens e sentimentos - ou externas - outros eventos vivenciados. A teoria do traço difuso (TTD) (Brainerd \& Reyna, 1995, 2005) propõe dois sistemas de memória distintos: a memória de essência (gist) e a memória literal (verbatim). Essa teoria pressupõe que as pessoas armazenam separadamente representações literais e de essência de uma mesma experiência, sendo as literais responsáveis pela captura dos detalhes específicos e superficiais, como por exemplo, "bebeu um guaraná", e as de essência são responsáveis pelo registro do significado da experiência, com o grau de generalidade podendo variar, como por exemplo, "bebeu um refrigerante". Assim, as taxas de esquecimento são diferentes para cada tipo de representação, e as memórias de essência se caracterizam por serem mais estáveis do que as literais ao longo do tempo (Brainerd \& Reyna, 2005). 


\section{PROCEDIMENTO DE PALAVRAS ASSOCIADAS (DRM) E TEMPO DE REAÇÃO}

Há diversas formas de estudar as falsas memórias: uso de frases livres em experimentos, histórias em prosa, sequência de diapositivos e vídeos. Porém, o procedimento mais utilizado nos últimos 18 anos é o procedimento Deese/ Roediger/ McDermott (DRM) (Roediger \& McDermott, 1995), ou procedimento de palavras associadas (Stein, Feix, \& Rohenkohl, 2006; Stein \& Perguer, 2001). Neste procedimento, os participantes estudam listas de palavras relacionadas com um tema que recebe a denominação de distrator crítico. Em testes de memória realizados imediatamente ou após algum tempo, os participantes reconhecem esse distrator crítico na forma de uma palavra intrusa ou mesmo evocam-no falsamente (Coane, McBride, Raulerson, \& Jordan, 2007).

A maioria dos estudos utilizando o procedimento DRM em tarefas de evocação ou reconhecimento constata o efeito robusto das FM utilizando dados de acurácia. Muito poucos estudos apresentam dados de tempos de reação (TR) no procedimento DRM (para exceções, ver Atkins \& Reuter-Lorenz, 2008; Coane et al., 2007; Jou, 2008; Jou, Matus, Aldridge, Rogers, \& Zimmerman, 2004; Tun, Wingfield, Rosen, $\&$ Blanchard, 1998). Isto por um lado é compreensível, pois o Zeitgeist científico da década de 1990 esteve voltado para as pesquisas que utilizaram o procedimento/ paradigma DRM (Roediger \& McDermott, 1995) em que a acurácia é a variável dependente principal. Por outro lado, o emprego do TR como variável dependente tem uma importância fundamental na história da psicologia (Galera \& Lopes, 1995; Welford, 1980) e em especial na psicologia cognitiva pelo estatuto ocupado por essa técnica durante os primórdios do paradigma do processamento de informação (Lachman, Lachman, \& Butterfield, 1979; Lopes, 1992; Lopes, Lopes, \& Teixeira, 2004). A vantagem de uma técnica cronométrica como esta é fornecer possibilidades de análises mais refinadas de processos cognitivos básicos, tais como a memória (Luce, 1986; Mc Nicol \& Stewart, 1980; Posner, 1978). A ideia básica do presente estudo é combinar o TR com o procedimento de palavras associadas em tarefas de curto prazo.

Um dos aspetos explicativos da ausência de estudos empregando o TR na medida de FM é que esse fenómeno tem sido estudado focalizando a memória episódica de longo prazo, envolvendo material verbal, que pode ser distorcido em intervalos de tempo que variam de semanas a meses (Atkins \& Reuter-Lorenz, 2008). Outro aspeto que merece atenção é que o aparecimento das falsas memórias no procedimento DRM se deve, entre outros fatores, ao grau de associação semântica do item crítico em relação aos itens da lista. A questão é se as associações semânticas podem ter efeitos em tarefas de curto prazo, e isso justificaria os estudos de FM 
em intervalos de tempo curtos. Estudos neuropsicológicos (Martin \& Romani, 1994; Martin \& Saffran, 1992), bem como outros modelos que incorporam a noção de códigos múltiplos e diferentes tipos de representação (Schneider \& Detweiler, 1987), sustentam a possibilidade de que os aspetos semânticos de uma informação podem estar acessíveis em diferentes tempos de processamento, ainda que muito rápidos. Além disso, evidências de categorização semântica na memória de curto prazo também foram obtidas em outros experimentos (Bartha, Martin, \& Jensen, 1998; Haarmann \& Usher, 2001; Jones \& Anderson, 1982). Em termos das teorias de FM, é preciso lembrar que na TTD os processos literais (verbatim) e os não literais (gist) operam tanto na memória de curto quanto na memória de longo prazo (Brainerd \& Reyna, 1990, 1992).

Do nosso conhecimento a partir de uma pesquisa feita na PsychINFO, com os termos-chave memory scanning, memory search e Sternberg's task, cruzando com falsas memórias ou falsa memória, no período de 1950 a 2010, encontrámos que um dos poucos trabalhos a empregar o TR nas FM é o de Coane et al. (2007). Ademais, foi o único estudo encontrado que empregou o paradigma experimental criado por Sternberg (1966) para estudar as FM utilizando listas DRM. Um estudo semelhante, porém no âmbito da memória de trabalho (working memory; Baddeley, 2007), foi realizado por Atkins e Reuter-Lorenz (2008). Coane et al. (2007) realizaram dois experimentos nos quais os sujeitos estudavam três, cinco ou sete itens de listas DRM e respondiam a um único estímulo (estudado ou não estudado). O primeiro experimento estava direcionado a saber se as FM poderiam ocorrer em tarefas de curto prazo, enquanto o experimento dois visava replicar o primeiro experimento e testar se os resultados obtidos nesse experimento eram resultados da diferença de itens, como por exemplo, a frequência deles. Em ambos os experimentos, os distratores críticos foram mais frequentemente reconhecidos do que os estímulos não apresentados de associação fraca. Além disso, a rejeição dos distratores críticos foi mais lenta do que a rejeição dos itens com fraca associação. Os dados foram interpretados à luz das teorias de ativação/ monitoramento (Roediger, Balota, \& Watson, 2001).

Partindo de pesquisas anteriores e da utilização do paradigma DRM nos estudos sobre o tema e considerando a importância das medidas de TR na psicologia cognitiva e a quase ausência desse tipo de medida nos experimentos com FM, o objetivo deste trabalho foi apresentar dados nesse contexto com a adaptação do DRM para o português brasileiro, denominado de pares associados (Stein et al., 2006; Stein \& Perguer, 2001), utilizando uma tarefa de reconhecimento de Sternberg (1966). Como hipóteses, espera-se (1) um aumento geral do TR em função do aumento do conjunto memorizado; (2) um aumento maior do tempo de reação na presença de FM e (3) um aumento do número de erros nas provas com a presença de FM. 


\section{MÉTODO}

\section{Participantes}

Este experimento contou com a participação voluntária de 12 sujeitos, sendo 9 do sexo masculino e 3 do sexo feminino $(M=23.8$ anos, $D P=3.45)$. Todos os participantes tinham visão normal ou corrigida e não apresentavam nenhuma demanda clínica.

\section{Material e estímulos}

Foram utilizadas as listas de palavras associadas adaptadas para o português do Brasil por Stein et al. (2006). As listas de palavras associadas são compostas por palavras tematicamente relacionadas, como por exemplo: escalada, natureza, beleza, árvores, liberdade, alpinismo, aventura, subir, rocha, russa, obstáculo, serra, terra, pico, morro. Estas palavras estão relacionadas ao tema "montanha". No entanto, esta palavra não está incluída na lista de palavras memorizadas, recebendo o nome de distrator crítico (DC). O DC é utilizado em uma tarefa de reconhecimento com o objetivo de observar a produção de uma falsa memória, caso o participante reconheça a sua apresentação na lista previamente memorizada. A partir destas listas padronizadas foram selecionados os conjuntos de palavras a serem memorizadas (CPM). Os CPM apresentavam três, cinco ou sete palavras (CPM 3, CPM 5 e CPM 7), conforme exemplificado na Figura 1.
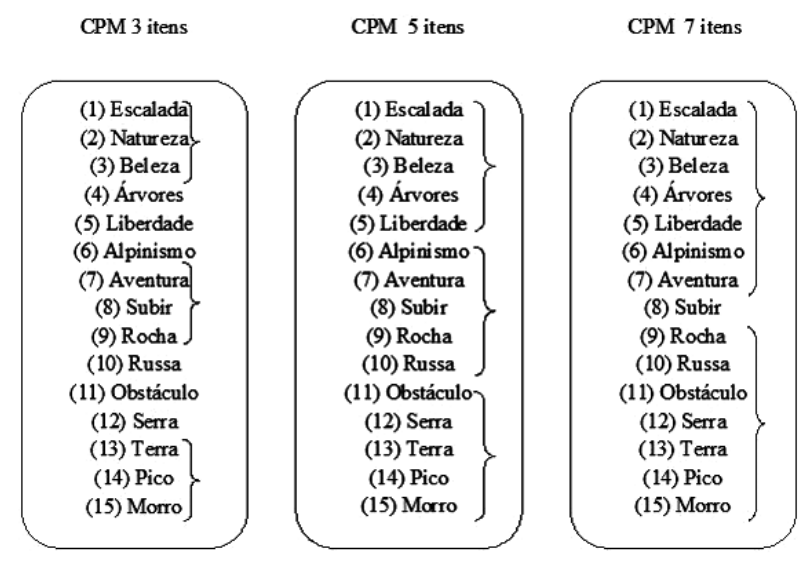

\section{Figura 1}

Exemplo do Processo de Seleção das Palavras para a Elaboração dos Conjuntos de Palavras Memorizadas (CPM). 
Os CPM foram selecionados com base em 39 das 44 listas de palavras associadas. As cinco listas remanescentes foram utilizadas para a construção das provas relativas aos distratores não relacionados (DNR). As palavras eram compostas por letras em fonte Arial 44 apresentadas na cor preta sobre um fundo branco.

As palavras que compunham cada CPM foram apresentadas serialmente na tela de um computador (2 MB de memória RAM, processador de $2.1 \mathrm{GHz}, \mathrm{HD}$ de 160 MB, em um monitor CRT - SyncMaster 794 Samsung ${ }^{\circledast}$ ). A apresentação dos estímulos e o registro das respostas dos participantes (tempo de reação e acurácia) foram executados pelo aplicativo E-Prime 2.0 Professional $^{\circledR}$ (Schneider, Eschman, \& Zuccolotto, 2002), em um ambiente reservado à realização da tarefa.

\section{Procedimento}

Os participantes realizaram o experimento durante uma única sessão experimental, de aproximadamente 45 minutos, subdividida em três blocos de provas realizados individualmente. A tarefa foi realizada em uma sala reservada com a luminosidade controlada. Todos os procedimentos adotados no presente estudo foram aprovados junto ao Comitê de Ética da Universidade Federal de Uberlândia (Protocolo 446/10).

Os participantes foram posicionados confortavelmente a uma distância de 60 $\mathrm{cm}$ do monitor sem nenhuma restrição aos movimentos. Eles foram instruídos a memorizar as palavras apresentadas em três blocos de provas apresentados em uma sequência pré-determinada, na qual metade dos participantes começou a tarefa pelo CPM 3 e a outra metade pelo CPM 7. O primeiro bloco (CPM 3) era composto por 36 provas. Após um intervalo de 60 segundos para descanso, o segundo bloco (CPM 5) era iniciado com a apresentação de 36 provas, seguido de um novo intervalo de 60 segundos para descanso. Após esse intervalo, o último bloco de provas, composto por 30 provas (CPM 7), era apresentado.

A tarefa do participante era memorizar as palavras apresentadas. Após a apresentação da série de palavras, uma palavra (teste) era apresentada para reconhecimento. Esta palavra poderia ter sido previamente apresentada (alvo) ou não (distrator crítico ou distrator não relacionado). Caso a palavra apresentada fosse reconhecida como uma palavra pertencente ao conjunto memorizado, o participante deveria pressionar a tecla "1" do teclado numérico. Caso a palavra apresentada não fosse reconhecida como uma palavra memorizada, o participante deveria pressionar a tecla " 2 " do teclado numérico.

Cada prova era iniciada com a apresentação de uma tela de descanso com a seguinte instrução "Pressione uma tecla para iniciar a prova”. Imediatamente após pressionar uma tecla, um ponto de fixação era apresentado no centro da tela por 1000 ms (sinal de soma “+” apresentado em fonte Arial 44). Após a apresentação 
do ponto de fixação, nenhum estímulo foi apresentado no intervalo de $250 \mathrm{~ms}$. Após este intervalo, a primeira palavra do CPM era apresentada no centro da tela por $750 \mathrm{~ms}$ seguidos de um intervalo de $250 \mathrm{~ms}$ no qual nenhum estímulo era apresentado. A seguir, a segunda palavra do CPM era apresentada por $750 \mathrm{~ms}$, e assim sucessivamente. Após a apresentação da última palavra do CPM, um arranjo de cinco sinais de asterisco posicionados lado a lado era apresentado por $750 \mathrm{~ms}$ com a função de máscara visual. Seguia-se uma palavra (teste) para reconhecimento que permanecia na tela até à resposta do participante. Imediatamente após a resposta, uma mensagem de resposta correta ou incorreta era apresentada por $500 \mathrm{~ms}$ encerrando a prova. A Figura 2 mostra uma representação esquemática da sequência de eventos que compunham uma prova experimental.

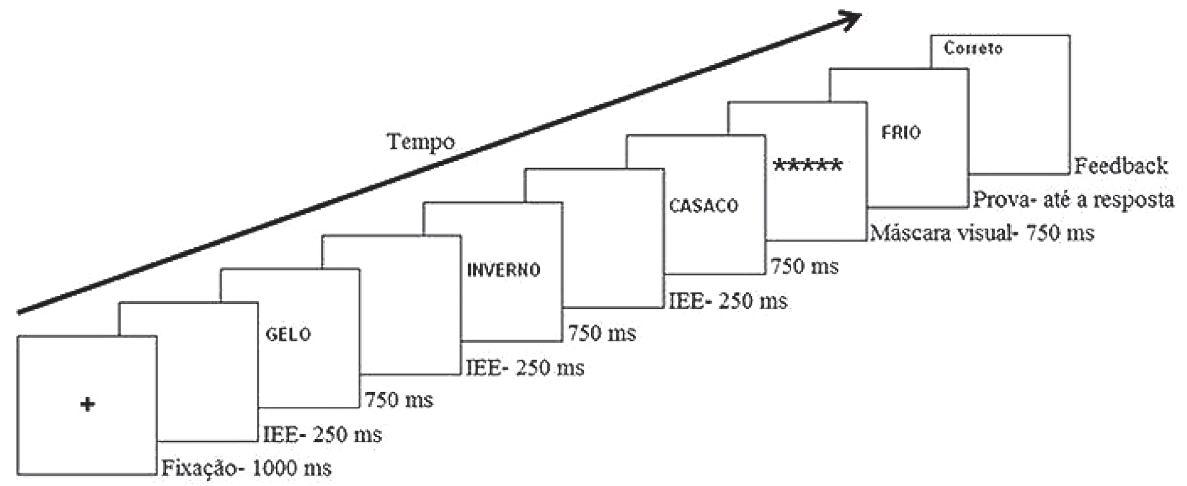

Figura 2

Representação Esquemática da Sequência de Eventos em uma Prova Experimental com a Apresentação de um Distrator Crítico (FRIO) e Extensão 3 (CPM 3). IEE = Intervalo Entre Estímulos.

Ao todo, cada participante respondeu a 102 provas (36 provas CPM 3, 36 CPM 5, 30 CPM 7). O bloco CPM 3 foi composto por 12 provas em que o teste pertencia ao conjunto memorizado (alvo), 12 provas em que o teste não pertencia ao conjunto memorizado, mas era relacionado semanticamente às palavras apresentadas (DC) e 12 provas em que o teste não pertencia ao conjunto memorizado e não mantinha relação semântica com as palavras apresentadas (DNR). A mesma proporção de tipos de prova foi utilizada no segundo bloco de provas (CPM 5). No terceiro bloco de provas (CPM 7) foram apresentadas 10 provas alvo, 10 provas DC e 10 provas DNR. Os participantes foram instruídos a ser rápidos e precisos em suas respostas. 


\section{RESULTADOS}

\section{Análise dos Tempos de Reação}

Os TR médios (ver Figura 3) para as respostas corretas (reconhecimento do alvo e rejeição correta das provas DNR e DC) foram submetidos à análise de variância para medidas repetidas (ANOVA). Foram excluídas da análise as provas com TR superior a dois desvios-padrão do tempo médio do participante na condição e os TR inferiores a $300 \mathrm{~ms}$.

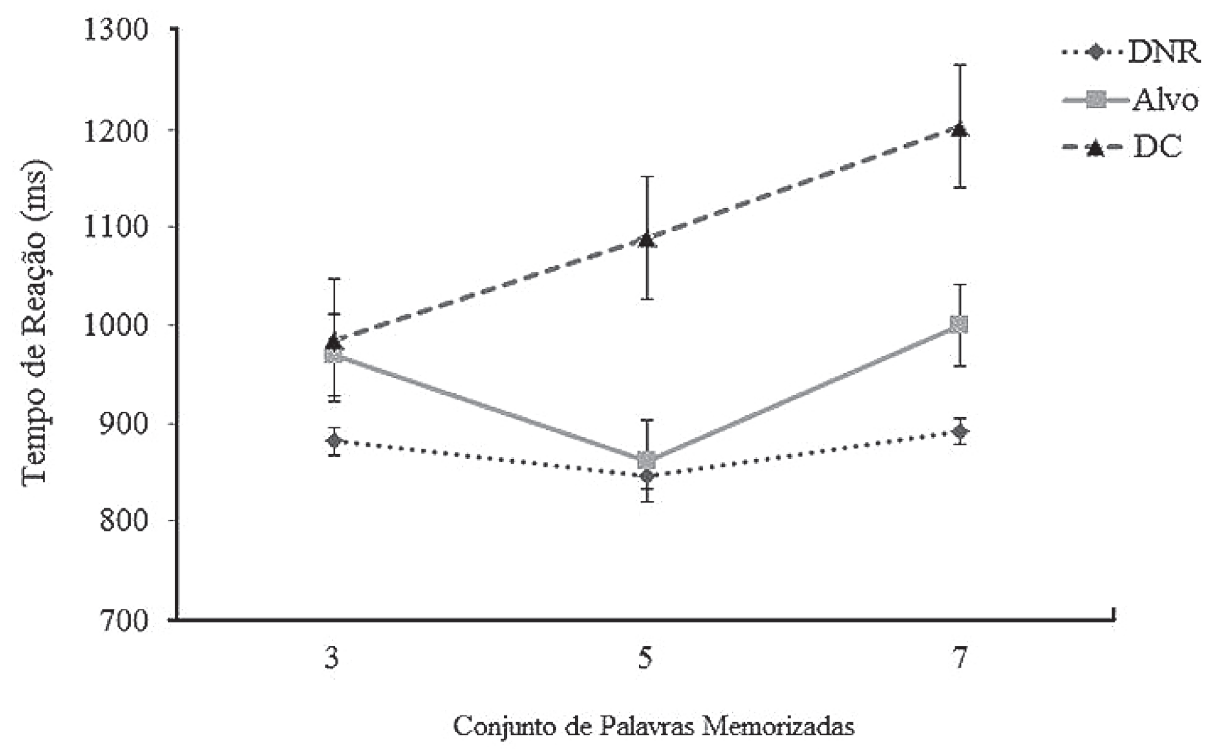

\section{Figura 3}

Média dos Tempos de Reação Obtidos nas Provas com Distrator Não Relacionado (DNR), Alvo, Distrator Crítico (DC) em Função do Conjunto de Palavras Memorizadas 3, 5, 7.

A análise considerou dois fatores principais: Conjunto de Palavras Memorizadas (CPM) (três, cinco, e sete palavras), X Tipo de Prova (TP) (distrator não relacionado (DNR), alvo, distrator crítico (DC). Esta análise confirmou um efeito significativo do fator $\operatorname{TP} F(2,22)=4.23, p<.05, \eta_{\mathrm{p}}^{2}=.28$, porém não evidenciou um efeito significativo do fator $\mathrm{CPM}, F(2,22)=1.44, p=.25$. Também não houve interação significativa entre os fatores investigados, $F(2,22)=1.76, p=.15$. 


\section{Análise dos erros}

A porcentagem de erros cometidos (ver Figura 4) foi submetida a uma ANOVA para medidas repetidas, considerando os mesmos fatores investigados para o TR. Esta análise confirmou um efeito significativo do fator CPM, $F(2,22)=11.3, p<.05, \eta_{\mathrm{p}}^{2}=.50$, bem como do fator TP, $F(2,22)=9.19 ; p<.05, \eta_{\mathrm{p}}^{2}=.45$. Houve interação significativa entre os fatores investigados, $F(4,44)=4.03, p<.05, \eta_{\mathrm{p}}^{2}=.27$, conforme mostra a Figura 4 .

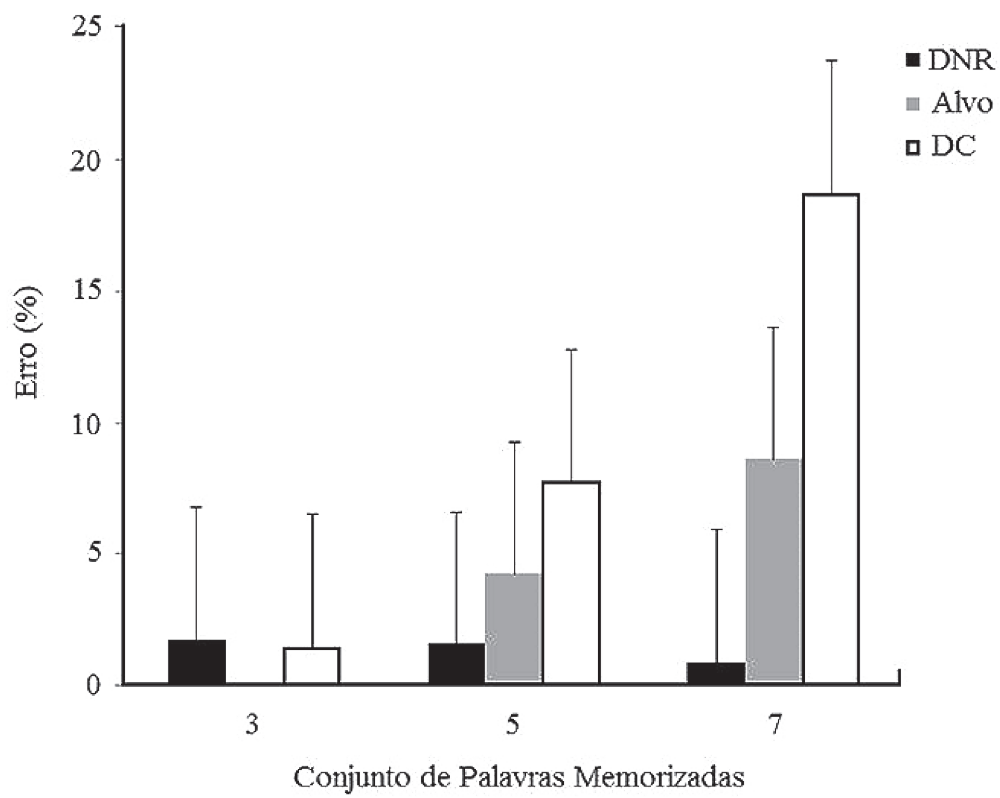

Figura 4

Porcentagem de Erros Cometidos nas Provas com Distrator Não Relacionado (DNR), Alvo, Distrator Crítico (DC) em Função da Extensão do Conjunto de Palavras Memorizadas 3, 5, 7.

O fator conjunto de palavras memorizadas foi analisado através do teste post hoc LSD $(\alpha=.05)$. Tal análise evidenciou uma porcentagem significativamente maior de erros cometidos nas provas CPM 7 em comparação com as provas CPM 3 ( $p=$ $.01)$ e CPM $5(p=.01)$. Não foi confirmado um aumento significativo do número de erros cometidos nas provas CPM 5 em comparação com as provas CPM $3(p=.06)$. A análise post hoc do fator TP confirmou uma diferença significativa na porcentagem de erros entre as provas alvo e distrator crítico $(p=.01)$ e distrator não relacionado e distrator crítico $(p=.01)$. Não houve diferença significativa na porcentagem de erros entre as condições de prova alvo e distrator não relacionado $(p=.13)$. 


\section{DISCUSSÃO}

Em linhas gerais, este estudo seguiu os mesmos parâmetros metodológicos de Coane et al. (2007). Do mesmo modo, toda a análise dos dados foi feita seguindo a lógica daqueles autores, portanto combinando as listas DRM (palavras associadas) com o procedimento experimental proposto por Sternberg (1966). Os dados obtidos são promissores: observa-se uma tendência de aumento do TR em função do conjunto de palavras memorizadas, embora a estatística não confirme esse resultado. Mas, de forma significativa, o TR é mais lento na rejeição de FM e produz mais erros nessa condição.

Os resultados obtidos mostram a robustez do fenômeno das FM, a despeito de algumas diferenças deste estudo com o de Coane et al. (2007). Coane et al. (2007) utilizaram o teste de reconhecimento a partir das 36 listas de Stadler, Roediger e McDermott (1999) sendo que foram utilizados quatro tipos de provas que variaram em força associativa em três diferentes tamanhos de conjuntos. Cada participante realizava 144 julgamentos divididos em 4 blocos, e cada bloco continha 12 conjuntos de cada tipo de tamanho, o que permitiu que cada tipo de prova fosse apresentado nove vezes.

No presente estudo, o delineamento experimental envolveu também o teste de reconhecimento, bem como os três tamanhos dos conjuntos utilizados por Coane et al. (2007), porém foram utilizados somente 3 tipos diferentes de provas. As listas utilizadas no presente estudo são provenientes da versão brasileira do procedimento DRM, e todas as 44 listas foram utilizadas, sendo 12 listas compondo o CPM 3, outras 12 compondo o CPM 5 e 10 listas compondo o CPM 7. As demais listas foram utilizadas como banco de palavras para utilização dos distratores não relacionados. Portanto, não foi possível controlar a força associativa entre as palavras, pois todos os conjuntos foram utilizados, uma vez que experimentos com tempo de reação exigem muitas provas, diferentemente de experimentos que testam unicamente acertos numa prova de evocação ou reconhecimento. O estudo de Coane et al. (2007) contou com uma amostra maior que aquela aqui apresentada. No entanto, em experimentos com TR é comum o emprego de pequenas amostras, pois em geral utiliza-se um planejamento de medidas repetidas, sendo importante a quantidade de provas mais do que o número de participantes. A favor desse argumento pesa também o tamanho dos efeitos das variáveis, que satisfazem a um critério desejável (Cohen, 1988).

Apesar dessas diferenças, os resultados obtidos neste estudo confirmam a possibilidade e a importância do TR como uma medida para se estudarem as FM com as listas de palavras associadas, em situações de curto prazo, a exemplo do trabalho de Coane et al. (2007). Sabe-se que o procedimento DRM produz fortes 
efeitos de FM, e esses efeitos ocorrem tanto nas tarefas de reconhecimento quanto nas tarefas de evocação. Dados obtidos no Brasil, e fora dele também, confirmam esses efeitos em diferentes experimentos (e.g., Alves \& Lopes, 2007; Gallo, 2010; Huang \& Janczura, 2008, 2013; Johanson \& Stenberg, 2002).

Parece-nos importante salientar que, a despeito do procedimento experimental com o paradigma DRM, as interpretações para os resultados em experimentos de curto prazo ainda dependem de mais investigação, sobretudo empregando a técnica do TR. Esta é uma medida mais sensível que pode captar as associações de forma mais fina e pode lançar luz sobre processos subjacentes às FM não captadas pela análise de desempenho dos participantes nesses experimentos. Uma análise de acordo com a proposta de Sternberg (1966), em termos de processamento serial versus paralelo e processamento exaustivo versus autoterminativo, permitiria um refinamento dos dados obtidos. Estamos a desenvolver atualmente um projeto de pesquisa nesse sentido. Uma análise desse tipo no experimento aqui apresentado só poderia ser feita com muita cautela. $O$ fato principal é que alguns procedimentos deveriam ser seguidos para permitir uma análise fidedigna das questões ligadas à forma do processamento. Talvez a principal seja o controle das respostas positivas (no atual caso representadas pelas respostas Alvo, ocupando 33\% das respostas) e das respostas negativas (respostas DNR e DC, ocupando 66\% das respostas). $\mathrm{Na}$ tarefa de Sternberg, essas respostas, em geral, são balanceadas, com 50\% das respostas positivas e 50\% negativas. Um outro aspeto com o qual se defronta nas tarefas envolvendo as palavras associadas é que a construção das listas de palavras depende de estudos de normatização, o que impede a escolha de quaisquer palavras. Isso limita o universo de palavras. Assim, o controle de posição serial, importante nos estudos de memória em geral, fica prejudicado nesse tipo de estudo, pois a repetição de uma palavra mudando apenas sua posição dentro de várias listas, mesmo que de diferentes tamanhos, poderia gerar efeitos de aprendizagem, o que fez com que gerássemos apenas uma lista com cada tipo de palavra diferente, considerando os 3 conjuntos utilizados. Uma possibilidade seria utilizar grupos independentes, mas isso poderia acarretar outras perdas em termos estatísticos quando se utiliza o TR. Apesar da nossa análise no presente estudo ter sido pensada segundo a proposta seguida em Coane et al. (2007), fizemos uma análise preliminar e superficial em termos do método de Sternberg (1966), a qual mostrou que, embora estatisticamente não significativa, há uma tendência de aumento do TR em função do aumento do conjunto de palavras, parecendo repetir o padrão observado na vasta literatura produzida a partir dos experimentos de Sternberg. No entanto, essa conclusão demanda ampliação das análises estatísticas, fazendo um estudo mais aprofundado com análises de regressão. Assim, poder-se-ia verificar se o aumento do TR é linear ou não, o que sugeriria o tipo de processamento predominante (serial ou 
paralelo). Muito embora, mesmo em condições controladas, haja diversos fatores que restringem uma escolha exclusiva entre tipos de processamento (Townsend, 1990). Outro aspeto interessante desta análise preliminar dos TR médios mostra que a relação entre as respostas "não" (respostas ao distrator crítico e ao distrator não relacionado) e as respostas "sim" (alvo) é maior que 1, sugerindo um possível processo de rechecagem das provas "não". Esse processo de rechecagem pode ser devido, em parte, ao fato de os distratores críticos (FM) não estarem presentes no conjunto memorizado, mas estão semanticamente relacionados às palavras que o compõem. Esse dado é bastante interessante em termos teóricos, pois ele pode ser adequadamente explicado, de forma parcimoniosa, pela teoria de ativação/monitoramento (Roediger et al., 2001). Roediger et al. (2001) apontam que uma interpretação é que a ativação de múltiplas palavras presentes em uma lista converge e inicia a palavra crítica não apresentada. Assim, se um alto grau de convergência produzir muita ativação para uma palavra crítica (distrator crítico) que não foi apresentada como para palavras da lista que foram estudadas atualmente, e se a ativação se mantiver durante parte da evocação e reconhecimento, então se pode utilizar a metáfora da ativação espalhada para se entender esse fenômeno.

Tanto os resultados de Coane et al. (2007) como os aqui apresentados parecem dar suporte para essa teoria, uma vez que as rejeições corretas e os falsos alarmes para os distratores críticos são mais lentos do que em outras condições. Deste modo, com maior ativação, a familiaridade para o distrator crítico aumenta e requer maior monitoramento do que palavras não relacionadas ou que têm um relacionamento fraco. De acordo com os autores, o aumento do tempo de processamento é aparente até para condições em que o conjunto é de três palavras, quando a precisão deve ser mais alta. O processo de monitoramento deve levar mais tempo quando da checagem das palavras em conjuntos maiores, o que pode ser constatado nas interações do conjunto de palavras memorizadas $\mathrm{x}$ tipo de prova, tanto na análise dos TR quanto dos erros. Também é importante lembrar que esse processo de rechecagem parece não ter sido suficiente para a rejeição dos distratores críticos, principalmente quando o conjunto de palavras memorizadas era composto por sete palavras. Os erros aqui subiram de forma alarmante, acompanhados de um aumento também no tempo de reação.

\section{CONCLUSÕES}

O presente estudo permitiu observar que o procedimento de palavras associadas adaptado a tarefas de curto prazo produz o mesmo efeito robusto das falsas 
memórias encontradas nas tarefas típicas de evocação a longo prazo. Os processos de monitoramento devem levar mais tempo quando se verificam itens num conjunto de memória maior na presença de distratores críticos (falsas memórias).

Em síntese, os mesmos processos presentes na produção de FM, medidos por outros meios, são passíveis de análise por meio do TR, embora análises mais refinadas ainda possam ser feitas com dados de TR e que foram exploradas aqui de forma preliminar.

\section{REFERÊNCIAS}

Alves, C. M., \& Lopes, E. J. (2007, Outubro). Efeitos da modalidade de apresentação das palavras e da instrução para o monitoramento da fonte na criação e persistência das falsas memórias. Trabalho apresentado na XXXVII Reunião Anual da Sociedade Brasileira de Psicologia. Florianópolis, SC, Brasil.

Atkins, A. S., \& Reuter-Lorenz, P. A. (2008). False working memory? Semantic distortion in a mere 4 seconds. Memory \& Cognition, 36, 74-81.

Baddeley, A. (2007). Working memory, thought, and action. Oxford: Oxford University Press.

Bartha, M. C., Martin, R. C., \& Jensen, C. R. (1998). Multiple interference effects in short-term recognition memory. American Journal of Psychology, 111, 89-118.

Bartlett, F. C. (1932). Remembering: A study in experimental and social psychology. New York: Cambridge University Press.

Brainerd, C. J., \& Reyna, V. F. (1990). Gist is the grist: Fuzzy-trace theory and the new intuitionism. Developmental Review, 10, 3-47.

Brainerd, C. J., \& Reyna, V. F. (1992). Explaining "memory free" reasoning. Psychological Science, 3, 332-339.

Brainerd, C. J., \& Reyna, V. F. (1995). Fuzzy-trace theory: Some foundational issues. Learning and Individual Differences, 7, 145-162.

Brainerd, C. J., \& Reyna, V. F. (2005). The science of false memory. New York: Oxford University Press.

Coane, J. H., McBride, D. M., Raulerson, B. III, \& Jordan, J. S. (2007). False memory in a short-term memory task. Experimental Psychology, 54(1), 62-70.

Cohen, J. (1988). Statistical power analysis for the behavioural sciences $\left(2^{\text {nd }}\right.$ ed.). New York: Academic Press.

Easenkraemer, R. E. (2006). Nas cercanias das falsas memórias. Ciências \& Cognição, 9, 97-110.

Galera, C., \& Lopes, E. J. (1995). Cronometria de processos mentais. Temas em Psicologia da SBP, 3, 1-10.

Gallo, D. (2010). False memories and fantastic beliefs: 15 years of the DRM illusion. Memory \& Cognition, 38, 833-848.

Haarmann, H., \& Usher, M. (2001). Maintenance of semantic information in capacity-limited item short-term memory. Psychonomic Bulletin \& Review, 8, 568-578.

Huang, T. P., \& Janczura, G. A. (2008). Processos conscientes e inconscientes na produção de falsas memórias. Psicologia: Teoria e Pesquisa, 24, 347-354.

Huang, T. P., \& Janczura, G. A. (2013). Contexto emocional negativo e processamento consciente na produção de falsas memórias em tarefas de reconhecimento. Psicologia: Reflexão e Crítica, 26, 534-542. 
Johanson, M., \& Stenberg, G. (2002). Inducing and reducing false memories: A Swedish version of the Deese-Roediger-McDermott paradigm. Scandinavian Journal of Psychology, 43, 369-383.

Johnson, M. K., Hashtroudi, S., \& Lindsay, D. S. (1993). Source Monitoring. Psychological Bulletin, $114,3-28$.

Jones, W. P., \& Anderson, J. R. (1982). Semantic categorization and high-speed scanning. Journal of Experimental Psychology: Learning, Memory \& Cognition, 8, 237-242.

Jou, J. (2008). Recall latencies, confidence, and output positions of true and false memories: Implications for recall and metamemory theories. Journal of Memory and Language, 58, 1049-1064.

Jou, J., Matus, Y. E., Aldrige, J. W., Rogers, D.M., \& Zimmerman, R. L. (2004). How similar is false recognition to veridical recognition objectively and subjectively? Memory \& Cognition, 32, 824-840.

Lachman, R., Lachman, J. L., \& Butterfield, E.C. (1979). Cognitive psychology and information processing: An introduction. Hillsdale, NJ: Lawrence Erlbaum.

Loftus, E. F. (1997). Creating false memories. Scientific American, 277, 70-75.

Loftus, E. F., \& Palmer, J. C. (1974). Reconstruction of automobile destruction: An example of the interaction between language and memory. Journal of Verbal Learning and Verbal Behavior, 13, 585-589.

Lopes, E. J. (1992). Codificação de sinais visuais na memória ativa evidenciada pelo método dos fatores aditivos. (Dissertação de mestrado não publicada). FFCLRP-USP, Ribeirão Preto, SP.

Lopes, E. J., Lopes, R. F. F., \& Teixeira, J. F. (2004). A psicologia cognitiva cinqüenta anos depois: A crise do paradigma do processamento de informação. Paidéia, 14, 17-26.

Luce, R. D. (1986). Response times: Their role in inferring elementary mental organization. New York: Oxford University Press.

Martin, R. C., \& Romani, C. (1994). Verbal working memory and sentence comprehension: A multiple components view. Neuropsychology, 8, 506-523.

Martin, N., \& Saffran, E. (1992). A computational account of deep dysphasia: Evidence from a single case study. Brain and Language, 32, 240-274.

Mazzoni, G. \& Scoboria, A. (2007). False memories. In F. T. Durso (Ed.) Handbook of applied cognition ( $2^{\text {nd }}$ edition, pp. 787-813). Chichester, UK:John Wiley \& Sons Ltd.

Mc Nicol, D., \& Stewart, G.W. (1980) Reaction time and the study of memory. In A.T. Welford (Ed.) Reaction times. New York: Academic Press.

Neufeld, C. B., Brust, P. G., \& Stein, L. M. (2010). Compreendendo o fenômeno das falsas memórias. In L. M. Stein et al., Falsas memórias: Fundamentos científicos e suas aplicações clínicas e jurídicas (pp. 21-41). Porto Alegre: Artmed.

Posner, M. I. (1978). Chronometric explorations of mind. Hillsdale, NJ: Lawrence Erlbaum.

Reyna, V. F., \& Lloyd, F. (1997). Theories of false memory in children and adults. Learning and Individual Differences, 9, 95-123.

Roediger, H. L., Balota, D. A., \& Watson, J. M. (2001). Spreading activation and arousal of false memories. In H. L. Roediger, J. S. Nairne, I. Neath, \& A . M. Surprenant (Eds.) The nature of remembering: Essays in honor of Robert G. Crowder (pp. 95-115). Washington, DC: APA Press.

Roediger, H. L. III, \& McDermott, K. B. (1995). Creating false memories: Remembering words not presented in lists. Journal of Experimental Psychology: Learning, Memory and Cognition, 21, 803-814.

Schneider, W., \& Detweiler, M. (1987). A connectionist/control architecture for working memory. In G. Bower (Ed.) The psychology of learning and motivation: Advances in research and theory (pp. 54-119). New York: Academic Press. 
Schneider, W., Eschman, A., Zuccolotto, A. (2002). E-Prime reference guide. Pittsburgh: Psychology Software Tools Inc.

Stadler, M. A., Roediger, H. L., \& McDermott, K. M. (1999). Norms for word lists that create false memories. Memory \& Cognition, 27, 494-500.

Stein, L. M., Feix, L. F., \& Rohenkohl, G. (2006). Avanços metodológicos no estudo das falsas memórias: Construção e normatização do procedimento de palavras associadas. Psicologia: Reflexão e Crítica, 19, 166-176.

Stein, L. M., \& Neufeld, C. B. (2001). Falsas memórias: Porque lembramos de coisas que não aconteceram?. Arquivos de Ciências da Saúde, UNIPAR, 5, 179-186.

Stein, L. M., \& Perguer, G. K. (2001). Criando falsas memórias em adultos por meio de palavras associadas. Psicologia: Reflexão e Crítica, 14, 353-366.

Sternberg, R. J. (2008). Psicologia cognitiva (4a ed.). Porto Alegre: Artes Médicas.

Sternberg, S. (1966). High speed scanning in human memory. Science, 153, 652-654.

Townsend, J. T. (1990). Serial vs. parallel processing: Sometimes they look like Tweedledum and Tweedledee but they can (and should) be distinguished. Psychological Science, 1(1), 46-54.

Tun, P. A., Wingfield, A., Rosen, M. J., \& Blanchard, L. (1998). Response latencies for false memories: Gist-based processes in normal aging. Psychology and Aging, 13, 230-240.

Welford, A. T. (1980). Reaction times. New York: Academic Press. 